\title{
Application of 7.0 T ultra-high-field MRI in evaluating the structure and function of the right ventricle of the heart in rats under a chronic hypoxic environment at high altitude
}

\author{
Yanqiu Sun ${ }^{1 \#}$, Chenhong Zhang ${ }^{1 \#}$, Dengfeng Tian ${ }^{1}$, Junhu Bai ${ }^{1}$, Yaodong Li $^{1}$, Xiaosheng Yu ${ }^{1}$, Jing Yang ${ }^{1}$, \\ Xueling Wang ${ }^{1}$, Yongxing Dong ${ }^{1}$, Mei Yang ${ }^{1}$, Zhiqiang Kang ${ }^{1}$, Qiang Zhang ${ }^{2}$, Fabao Gao \\ ${ }^{1}$ Department of Radiology, Qinghai Provincial People's Hospital, Xining, China; ${ }^{2}$ Department of Neurosurgery, Qinghai Provincial People's \\ Hospital, Xining, China; ${ }^{3}$ Department of Radiology, West China Hospital of Sichuan University, Chengdu, China \\ Contributions: (I) Conception and design: Y Sun, C Zhang, Q Zhang, F Gao; (II) Administrative support: Q Zhang, F Gao; (III) Provision of study \\ materials or patients: Y Sun, F Gao, D Tian, J Bai, Y Li, X Yu; (IV) Collection and assembly of data: C Zhang, D Tian, J Yang, X Wang, Y Dong, M \\ Yang; (V) Data analysis and interpretation: C Zhang, D Tian, J Bai, Y Li, X Yu, J Yang, X Wang, Z Kang; (VI) Manuscript writing: All authors; (VII) \\ Final approval of manuscript: All authors. \\ "These authors contributed equally to this work and should be considered as co-first authors. \\ Correspondence to: Dr. Qiang Zhang. Department of Neurosurgery, Qinghai Provincial People's Hospital, Xining, China. \\ Email: zhangqiang691212@163.com; Dr. Fabao Gao. Department of Radiology, West China Hospital of Sichuan University, Chengdu, China. \\ Email: gaofabao@wchscu.cn.
}

Background: Long-term exposure to a high-altitude environment with low pressure and low oxygen can cause abnormalities in the structure and function of the heart, in particular the right ventricle. Monitoring the structure and function of the right ventricle is therefore essential for early diagnosis and prognosis of high-altitude heart-related diseases. In this study, 7.0 T MRI is used to detect cardiac structure and function indicators of rats in natural plateau and plain environments.

Methods: Rats in two groups were raised in different environments from 6 weeks of age for a period of 12 weeks. At 18 weeks of age both groups underwent $7.0 \mathrm{~T}$ cardiac magnetic resonance (CMR) scanning. Professional cardiac post-processing software was used to analyze right ventricular end-diastolic volume (RVEDV), right ventricular end-systolic volume (RVESV), right ventricular stroke volume (RVSV), right ventricular ejection fraction (RVEF), Right ventricular end-diastolic myocardial mass (RV Myo mass, diast), Right ventricular end-systolic myocardial mass (RV Myo mass, syst), tricuspid valve end-diastolic caliber (TVD), tricuspid valve end-systolic caliber (TVS), right ventricular end-systolic long-axis (RVESL) and right ventricular end-diastolic long-axis (RVEDL). Prior to the CMR scan, blood was collected from the two groups of rats for evaluation of blood indicators. After the scan, the rats were sacrificed and the myocardial tissue morphology observed under a light microscope.

Results: In the group of rats subject to chronic hypoxia at high altitude for 12 weeks (the plateau group), red blood cell (RBC) count, hemoglobin (HGB) and hematocrit (HCT) increased $(\mathrm{P}<0.05)$; RVEDV, RVESV, RVSV, RV Myo mass (diast), RV Myo mass (syst), TVS, RVESL, and RVEDL also increased $(\mathrm{P}<0.05)$. Observation of the right ventricle of rats in the plateau group using a light microscope mainly showed a slightly widened myocardial space, myocardial cell turbidity, vacuolar degeneration, myocardial interstitial edema, vascular congestion and a small amount of inflammatory cell infiltration.

Conclusions: The importance of ultra-high-field MRI for monitoring the early stages of rat heart injury has been demonstrated by studying the changes in the structure and function of the right ventricle of rats subject to chronic hypoxia at high altitude over a period of 12 weeks.

Keywords: Plateau; hypoxia; SD rats; 7.0 T MRI; right ventricle 
Submitted Aug 31, 2021. Accepted for publication Oct 16, 2021.

doi: 10.21037/atm-21-5078

View this article at: https://dx.doi.org/10.21037/atm-21-5078

\section{Introduction}

Plateau areas are characterized by: low temperature, low humidity, large variations in temperature, and strong ultraviolet radiation (1). Among these, low pressure and low oxygen are the primary characteristics of plateau areas (2). The heart is very sensitive to hypoxia, and the pulmonary artery is a functional blood vessel connecting the heart and the lungs. Low-pressure and low-oxygen stimulation can cause pulmonary vasoconstriction and increase pulmonary vascular resistance, resulting in pulmonary hypertension. Pulmonary hypertension occurs when hypoxic stimulation causes remodeling of small arteries in the lungs that lack smooth muscle cells. Exposure to a high-altitude environment with low pressure and low oxygen can cause red blood cell proliferation and increase hematocrit $(3,4)$. An excessive increase in hematocrit will increase blood viscosity; this leads to increased microcirculatory resistance, slow blood flow, and the aggregation of red blood cells, thus affecting the transport of oxygen by blood $(4,5)$. Chronic hypoxia at high altitude will affect the myocardial structure and function of rats. With an increase in altitude and prolonged exposure time, the steady-state supply of oxygen is unbalanced, which gradually aggravates myocardial tissue damage (6). The effect of chronic hypoxia at high altitude on rat myocardial structure is mainly shown as right-sided hypoxic myocardial remodeling, which is clinically manifested as changes in cardiac volume, geometric shape, and cardiac function, and pathological features are myocardial cell hypertrophy and right ventricular hypertrophy and myocardial fibrosis $(7,8)$. Studies have shown that myocardial hypertrophy and myocardial fibrosis have different regulatory mechanisms, the increase in right ventricular afterload caused by hypoxic pulmonary hypertension and red blood cell proliferation is the main factor leading to cardiomyocyte hypertrophy and right ventricular hypertrophy, the interaction between cardiac fibroblasts and extracellular matrix during hypoxia is an important factor in inducing myocardial fibrosis (9-11). The effect of chronic hypoxia on rat myocardial function is manifested in that myocardial cells increase the maximum utilization of limited oxygen by increasing mitochondrial biosynthesis, and promote the conversion of metabolic substrates by regulating the activity of metabolicrelated kinases, increasing the utilization of glucose and reducing the utilization of fatty acids. And by increasing the expression of anti-apoptotic factors to enhance the ability of cardiomyocytes to withstand stress, the adaptation of these cardiomyocytes to the chronic low-pressure and hypoxic environment is collectively referred to as the myocardial chronic hypoxia response (12). However, when the myocardium is exceeded due to long-term severe chronic hypoxia When adaptability, it will cause reversible myocardial function inhibition (13). Other scholars believe that the high altitude hypoxic environment can stimulate sympathetic nerve excitement and repeatedly act on myocardial beta receptors, causing myocardial cell apoptosis and ventricular remodeling, which ultimately leads to heart failure (14). Studies showed that the initial cardiovascular response to altitude is characterized by an increase in cardiac output with tachycardia, no change in stroke volume. After a few days of acclimatization, cardiac output returns to normal, but heart rate remains increased, so that stroke volume is decreased, the index of right ventricular systolic function initially increases, then preserved or slightly depressed and an altered diastolic filling pattern. Whether this is explained by an improved maximum flow output by an unloaded right ventricle remains to be confirmed (15).

Early monitoring of the structure and function of the right ventricle is essential for early diagnosis and prognosis of high-altitude heart-related diseases. Two-dimensional (2D) transthoracic echocardiography (TTE) is the major non-invasive diagnostic tool for assessing heart structure and function, especially under field conditions as at high altitude (16). However, Cardiac Magnetic Resonance (CMR) imaging is the gold standard for evaluating myocardial function and measuring heart volume, and can simultaneously evaluate the structure and function of the heart through a "one-stop" noninvasive examination $(17,18)$. In this experiment, we used the 7.0 T CMR to analyze the changes in the structure and function of the right ventricle in rats under chronic hypoxic conditions at high altitude, and to provide imaging evidence for the mechanism of cardiac injury associated with chronic altitude sickness. We present the following article in accordance with the ARRIVE reporting checklist (available at https://dx.doi. 
org/10.21037/atm-21-5078).

\section{Methods}

\section{Rat}

SD rats (male, 6 weeks old, 31 in total) were purchased from Chengdu Dashuo Experimental Animal Co., Ltd. [Experimental Animal Production License Number: SCXK (Chuan) 2020-030, Experimental Animal License Number: SYXK (Chuan) 2018-119] and divided into two groups: 21 in the plateau group (Yushu, Qinghai, altitude $3,800 \mathrm{~m}$ ) and 10 in the plain group (Chengdu, Sichuan, altitude $500 \mathrm{~m}$ ). The number plate method was used to number the experimental animals, and the average body weight was $150.12 \mathrm{~g}$. The living environment of the rats was as follows: $12 \mathrm{~h}$ day and night alternation, clean grade, room temperature $18-25^{\circ} \mathrm{C}$, humidity $40-60 \%$, with free access to food and drinking water. All rats were raised to 18 weeks of age at the same time, after which the average body weight of the plateau group was $515.57 \mathrm{~g}$, the plain group was $501.20 \mathrm{~g}$, and approved by the Laboratory Animal Protection and Use Committee of West China Hospital of Sichuan University. The disposal of animals during the experiment complied with the Pain Management Standards in the Eighth Edition of "Guidelines for the Care and Use of Laboratory Animals" (19). Experiments were performed under a project license [No. SYXK ( Qing ) 2019-0001] granted by the ethics committee of Qinghai Provincial Department of Science and Technology, in compliance with the Drug Inspection and Testing in Qinghai Province institutional guidelines for the care and use of animals. A protocol was prepared before the study without registration.

\section{General physiological data collection}

The body weight of the rats in the plateau group and the plain group was measured and recorded before the cardiac MRI scan. The tail vein puncture blood collection method was used to collect rat blood; this required inhalation anesthesia, where the anesthetic gas was set to a mixture of $2 \%$ to $3 \%$ isoflurane and oxygen. After the rat was anesthetized the blood volume $(1-2 \mathrm{~mL})$ was collected using the venous trocar catheter for animal-specific venous blood collection with a vacuum blood collection tube. Hematological indices, including RBC, HGB and HCT count, were determined using a Coulter Automated Cell Counter (Coulter AcT, Beckman Coulter, New York, NY, USA) (20).

\section{Routine cardiac MRI scan and post-processing}

\section{Equipment}

A 7.0 T magnetic resonance scanner (Bruker BioSpec70/30USR, Ettlingen, Germany) from West China Hospital of Sichuan University was used. The imaging coil was composed of a $72-\mathrm{mm}$ birdcage radio frequency signal transmitting coil, and a 56-mm inner diameter array surface echo signal receiving coil. The SAII Model 1030 small animal monitoring/gating system (Small Animal Instruments, Inc., Stony Brook, NY, USA) was used to collect animal respiratory and electrophysiological parameters; rats were anesthetized throughout the whole scanning process with the MATRXVIP3000 small animal respiratory anesthesia machine (MIDMARK Company, USA).

\section{CMR scanning process}

Before the CMR scan, each rat was placed in a closed glass cylinder filled with a mixture of oxygen and isoflurane gas, where $2 \%$ to $3 \%$ isoflurane was used for inhalation anesthesia. After anesthesia was induced, the rat was immediately transferred to the scanning bed and a special mask fitted to continuously deliver inhalation anesthesia. The chest of the rat was positioned at the center of the coil, ECG electrodes were placed on both the forelimb and right hind limb, and a respiration sensor was placed on the abdomen. MRI scans were performed once breathing and ECG signals were stable, and data on the heart rate and respiration indices of the two groups of rats was collected.

A three-plane positioning sequence scan of the chest was performed, and the four-chamber heart, standard left ventricle two-chamber, left ventricle and right ventricle short-axis movie images were then collected with reference to the positioning image. The parameter settings were as follows: echo time (TE) $2.5 \mathrm{~ms}$, repetition time (TR) $8.00 \mathrm{~ms}$, flip angle (FA) $20^{\circ}$, layer thickness $1.5 \mathrm{~mm}$, layer spacing $0 \mathrm{~mm}$, field of view (FOV) $50 \mathrm{~mm} \times 50 \mathrm{~mm}$, and the matrix was $256 \times 256$. The number of excitations (NEX) was 4 , and 15 frames of film imaging were collected in each cardiac cycle. The total scanning time was 30-40 min.

\section{Image data processing and analysis}

The continuous short-axis, long-axis, two-chamber, and four-chamber DICOM images of the right ventricle were imported into the professional cardiac image processing software Circle CVI42 (Circle Cardiovascular Imaging Inc., Calgary, Alberta, Canada), and the software automatically recognized the end-diastolic and end-systolic stages. The contours of the right ventricle endocardium 
Table 1 Comparison of general physiological data for rats in the plateau group and the plain group

\begin{tabular}{|c|c|c|c|c|}
\hline Index & Plateau group $(n=21)$ & Plain group $(n=10)$ & $\mathrm{t}$ value & $P$ value \\
\hline Respiration rate (times/min) & $303.86 \pm 36.09$ & $318.70 \pm 23.59$ & -1.368 & 0.183 \\
\hline Heart rate (beats/min) & $40.38 \pm 5.55$ & $42.40 \pm 6.06$ & -0.919 & 0.365 \\
\hline RBC count $\left(10^{12} / \mathrm{L}\right)$ & $9.61 \pm 0.45$ & $8.81 \pm 0.48$ & 4.566 & $8.5 \mathrm{E}-5$ \\
\hline HCT (\%) & $53.90 \pm 2.16$ & $47.67 \pm 2.10$ & 7.576 & $<0.1 \mathrm{E}-5$ \\
\hline
\end{tabular}

RBC, red blood cell; HGB, hemoglobin; HCT, hematocrit.

and epicardium in these two phases were then manually traced, layer by layer. The CVI42 software SD short module was used to analyze right ventricular function to obtain the following functional and structural indicators: RVEDV, RVESV, RVSV, RVEF, RV Myo mass (diast), RV Myo mass (syst).

The TVS and RVESL were measured on the standard ventricular long-axis end-systolic four-chamber heart plane, while the TVD and RVEDL were measured on the standard ventricular long-axis end-diastolic four-chamber heart plane. These data measurements were performed by a professional physician, and where averages were obtained from three repeat measurements. After the scans the rats were sacrificed, and the myocardial tissue morphology observed under a light microscope.

\section{Pathological tissue sampling}

On the day after the MRI scan was completed, the rat was killed by injecting potassium chloride into the tail vein. The heart was removed and washed with saline, then the myocardial tissue was fixed in $10 \%$ formaldehyde, embedded in paraffin, sectioned and stained with conventional hematoxylin-eosin (HE). The pathological changes in the myocardial tissue of the right ventricle were observed under an optical microscope.

\section{Statistical analysis}

SPSS 23.0 statistical software was used for the analysis. The measurement data have been expressed as means \pm standard deviation. The independent sample $t$-test was used for parameter comparison between the two groups, where $\mathrm{P}<0.05$ indicated that the observed difference was statistically significant.

\section{Results}

\section{General physiological data for SD rats}

A comparison of the general data obtained for the rats (Table 1) revealed no significant difference in body weight, respiration rate or heart rate between the plateau group and the plain group $(\mathrm{P}>0.05)$. The red blood cells $(\mathrm{RBC})$ count, hemoglobin (HGB) and hematocrit (HCT) values of the plateau group were significantly higher than those of the plain group, and the difference was statistically significant $(\mathrm{P}<0.01)$.

\section{Cardiac MRI scan index}

Table 2 presented a quantitative comparison of the data characterizing the structure and function of the right ventricle in the plateau group and the plain group obtained from the imaging study (a representative set of images was shown in Figure 1). The parameter values of right ventricular end-diastolic volume (RVEDV), right ventricular end-systolic volume (RVESV), right ventricular stroke volume (RVSV), Right ventricular diastolic myocardial mass (RV Myo mass, diast), Right ventricular systolic myocardial mass (RV Myo mass, syst), tricuspid valve endsystole caliber (TVS), right ventricular end-systolic longaxis (RVESL) and right ventricular end-diastolic long-axis (RVEDL) were higher in the plateau group than in the plain group $(\mathrm{P}<0.05)$; there were no statistically significant differences in the tricuspid valve end-diastolic caliber (TVD) and right ventricular ejection fraction (RVEF) between the two groups, however the RVEF of rats in the plateau group was lower than in the plain group. Figure 2 presents these same results as a series of comparative bar charts that illustrate the observed trends. 
Table 2 Comparison of the structure and function of the right ventricle of rats in the plateau group and the plain group

\begin{tabular}{|c|c|c|c|c|}
\hline Index & Plateau group $(n=21)$ & Plain group $(n=10)$ & $\mathrm{t}$ value & $P$ value \\
\hline $\mathrm{RVESV} / \mathrm{mL}$ & $0.22 \pm 0.07$ & $0.13 \pm 0.04$ & 3.500 & 0.002 \\
\hline $\mathrm{RVSV} / \mathrm{mL}$ & $0.34 \pm 0.07$ & $0.25 \pm 0.06$ & 3.387 & 0.002 \\
\hline RVEF/\% & $61.34 \pm 6.75$ & $65.45 \pm 3.93$ & -1.176 & 0.086 \\
\hline RV Myo mass (syst)/g & $0.36 \pm 0.11$ & $0.22 \pm 0.05$ & 3.894 & 0.001 \\
\hline $\mathrm{TVS} / \mathrm{mm}$ & $2.80 \pm 0.59$ & $2.28 \pm 0.20$ & 3.598 & 0.001 \\
\hline RVESL/mm & $10.97 \pm 2.03$ & $9.02 \pm 1.74$ & 2.610 & 0.014 \\
\hline $\mathrm{TVD} / \mathrm{mm}$ & $4.02 \pm 0.71$ & $3.99 \pm 0.69$ & 0.059 & 0.953 \\
\hline
\end{tabular}

RVEDV, right ventricular end-diastolic volume; RVESV, right ventricular end-systolic volume; RVSV, right ventricular stroke volume; RVEF, right ventricular ejection fraction; TVS, tricuspid valve end-systole caliber; RVESL, right ventricular end-systolic long-axis; TVD, tricuspid valve end-diastolic caliber; RVEDL, right ventricular end-diastolic long-axis.
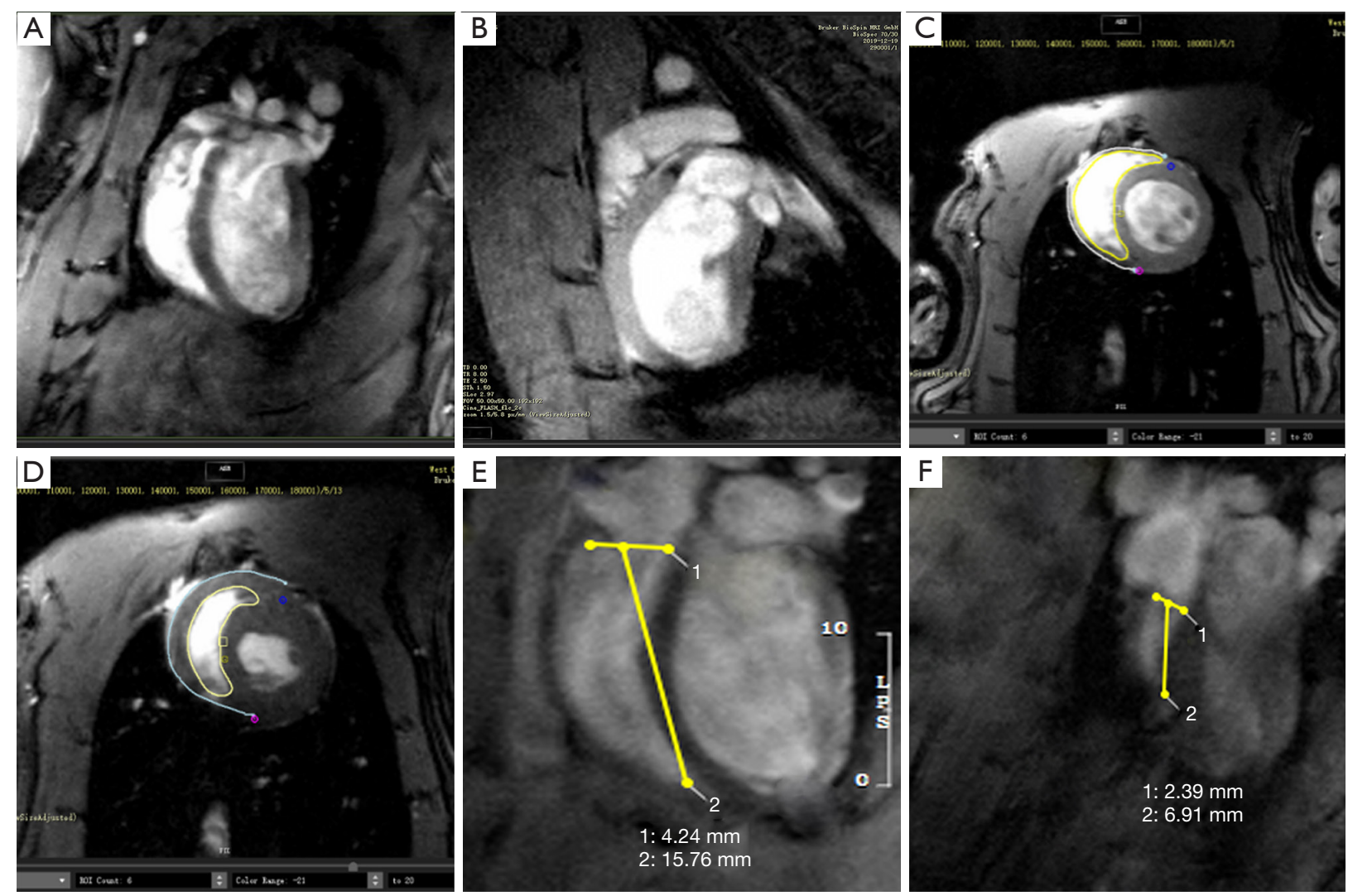

Figure 1 Heart scan and data measurement images for rats in the plateau group and the plain group. (A,B) Standard four-chamber heart and left ventricle two-chamber view; (C,D) right ventricular end-diastolic and end-systolic endo- and epicardial delineation images; (E,F) at the four-chamber heart level, the end-diastolic and end-systolic tricuspid valve calibers and the long-axis of the right ventricle were measured, denoted by 1 and 2 respectively. 
A
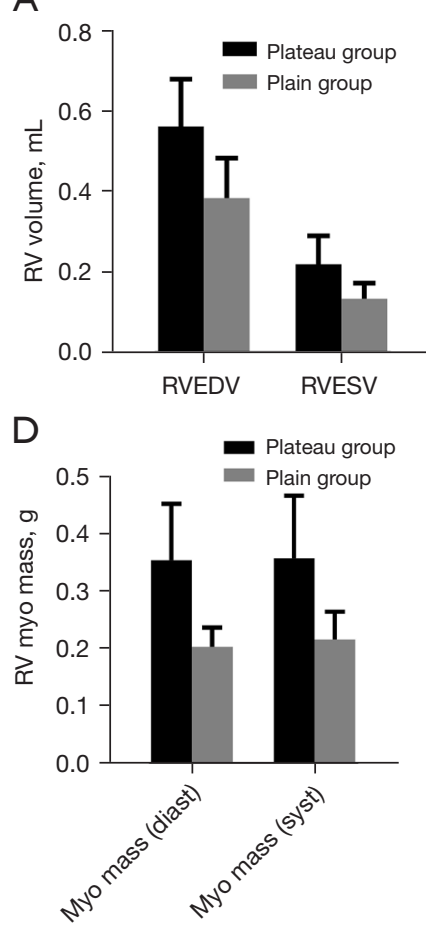

B

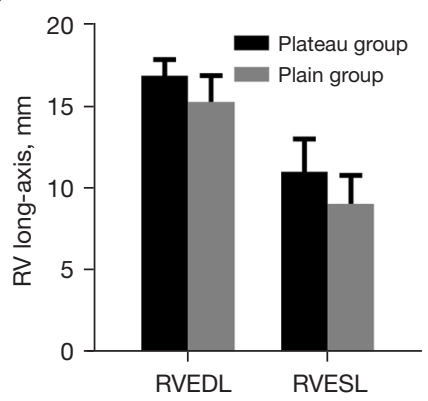

$\mathrm{E}$

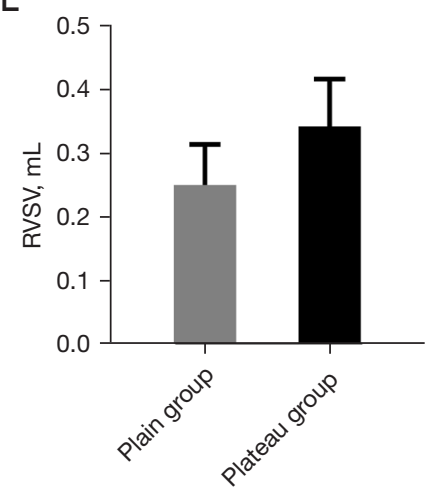

C

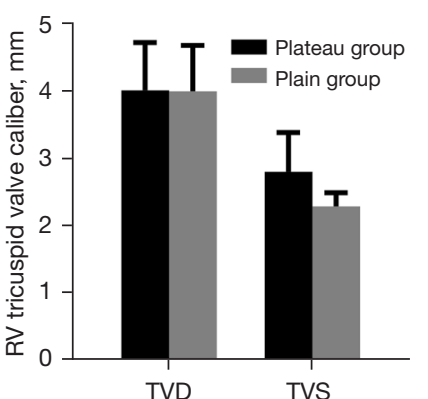

$\mathrm{F}$

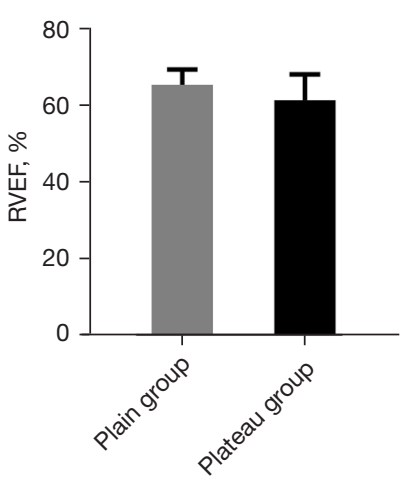

Figure 2 Comparison of the structure and function parameters of the right ventricle of rats in the plateau group and plain group. RVEDV, right ventricular end-diastolic volume; RVESV, right ventricular end-systolic volume; RVEDL, right ventricular end-diastolic long-axis. RVESL, right ventricular end-systolic long-axis; TVD, tricuspid valve end-diastolic caliber; TVS, tricuspid valve end-systole caliber; RVSV, right ventricular stroke volume; RVEF, right ventricular ejection fraction.

\section{Pathological results}

Light microscopy observation of the right ventricle of rats in the plateau group mainly showed a slightly widened myocardial space, myocardial cell turbidity, vacuolar degeneration, myocardial interstitial edema, vascular congestion and a small amount of inflammatory cell infiltration (see Figure 3). The right ventricular cardiomyocytes of the plain group showed no obvious abnormal changes in the morphological structure.

\section{Discussion}

When people enter the plateau from the plain, the basic physiological indexes such as heart rate and respiratory rate will change. When the partial pressure of arterial oxygen drops to $35-45 \mathrm{mmHg}$, the heart rate will increase by $40-50 \%$. Hypoxia leads to increased heart rate, respiratory rate, gas volume in and out of the alveoli, and gas exchange capacity to maintain the ventilation/blood flow (V/Q) ratio (21). The V/Q ratio is in the normal range, and an appropriate increase in heart rate can increase cardiac output, which is also a manifestation of the body's reserve capacity and compensatory function, which is a method of self-protection. The study by Zhao et al. (22) observed that acute exposure to a hypoxic environment causes the heart rate to increase and blood oxygen saturation to decrease. However as time passes, the heart rate does not continue to increase but returns to normal. For people who live in a plateau area, a drop in heart rate is also a physiological adaptation process of the body. By observing the basic indicators of the two groups of rats, it was found that the heart rate and respiration rate of rats in the plateau group were slightly lower than those in the plain group, however there was no significant difference. The results show that the rats adapted to the plateau environment after being continuously exposed for a period of 12 weeks.

A comparison of the general physiological indicators of the two groups of rats shows that the blood indicators HGB, RBC count, and HCT in the plateau group are significantly 

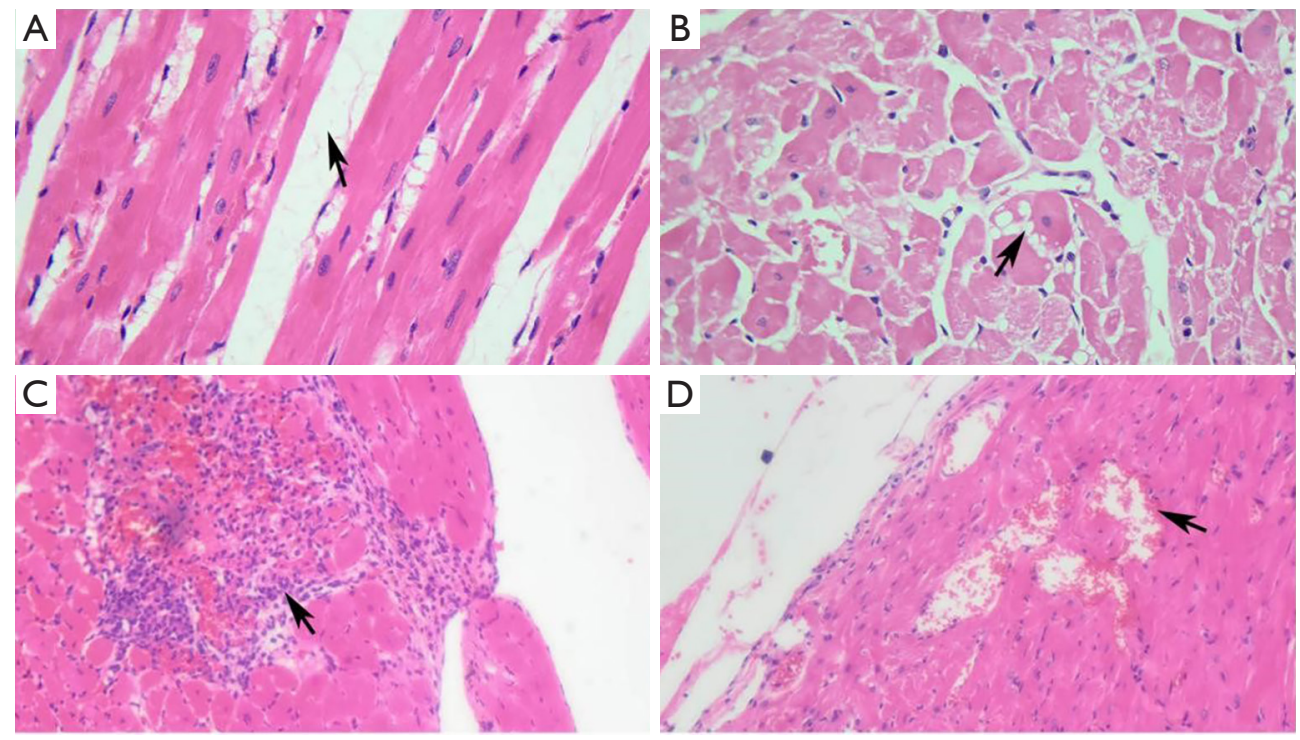

Figure 3 Pathology of the right ventricle in the plateau group: (A,B) show slightly widened myocardial space myocardial cell turbidity and vacuolar degeneration (black arrow, HE staining, $\times 40)$. (C,D) show myocardial interstitial edema, vascular congestion and inflammatory cell infiltration (black arrow, HE staining, $\times 10$ ).

higher than those in the plain group. Erythropoietin (EPO) is produced in the kidney and a small part of the liver. EPO is both a hormone and a glycoprotein. EPO is essential in the differentiation of red blood cells and has a protective effect on the body from hypoxia (23). Studies have shown that vascular endothelial growth factor (VEGF) plays a key role in the proliferation of endothelial cells and the formation of new blood vessels during hypoxia (24). Hypoxia causes myocardial damage and induces the secretion of VEGF, resulting in the formation of new blood vessels. The main pathological changes are myocardial fiber hyperplasia and interstitial blood vessel dilation, and hyperemia will eventually accelerate the production of new blood vessels and improve heart function (24). As the "guard" of cytokines, EPO expression speeds up when the body is hypoxic, and EPO expression is related to hypoxia inducible factors (HIFs). After entering a plateau area, the expression of HIFs in the cells is increased. The downstream EPO gene is activated, the synthesis and secretion of EPO increase, and the red blood cell count increases, which helps to improve tissue oxygen supply and is another compensatory response of the body to hypoxia. In related literature considering the chronic hypoxic environment of plateau regions (25), hypoxia is found to stimulate the synthesis and release of EPO and to promote the proliferation of red blood cells. Although the increase in red blood cells helps to improve the oxygen- carrying capacity of blood at high altitudes to a certain extent, an excessive number of red blood cells will lead to increased blood viscosity, increased blood flow resistance, decreased blood flow speed and increased heart workload. This is also a major contributor to myocardial damage caused by longterm high-altitude hypoxia.

The 7.0 T ultra-high-field MRI significantly improves the image signal-to-noise ratio and spatial resolution, and meets the needs of ultra-high resolution imaging and functional metabolic imaging. At present, in addition to monitoring the structure and function of the heart, it is also used for nerve, blood vessels, tumors and bones and joints. In this study, we used 7.0T MRI to study the changes in the function and structure of the right ventricle in rats at high altitude, the results of this study showed that RVEDV, RVESV, RVSV, RV Myo mass (diast), RV Myo mass (syst), TVS, RVESL and RVEDL in the plateau group were higher than in the plain group. Pathological analyses have found that the right ventricular myocardial space in the plateau group was slightly increased. Pathological changes, such as a slightly widened myocardial space, myocardial cell turbidity, vacuolar degeneration, myocardial interstitial edema, vascular congestion, and a small amount of inflammatory cell infiltration, may be caused by the damage of hypoxia itself to myocardial cells. Normally, the myocardium uses fatty acids as its main 
energy source, accounting for $60 \%$ to $70 \%$ of myocardial oxygen consumption, and glucose metabolism accounts for approximately $30 \%$. In chronic hypoxia, however, myocardial tissue glycogen content is reduced, glucose uptake and utilization are enhanced, and fatty acid oxidation is reduced (26). Nakada et al. $(9-11,27)$ observed myocardial cell hypertrophy and myocardial degeneration in the early stages of hypoxia, collagen fiber tissue proliferation, myocardial fiber enlargement and thickening in the later stages, and they found that these changes can cause heart remodeling. The earliest indication of heart remodeling is increased ventricular mass, which leads to further changes in the diameters of the heart's chambers and the volumes of the ventricles. A small amount of autopsy data in Tibet showed that the right ventricle was hypertrophic, the inner diameter of myocardial cells was enlarged, the cells were swollen, capillary density was increased, and mitochondria were increased in size under the microscope (25). This study also found that the right ventricular function and structural parameters of rats in the plateau group changed, and the RVEF tended to decrease, but still maintained a normal state. The reason may be related to the altitude exposure time and the reserve function of the heart. After 12 weeks of continuous exposure, right ventricular function is impaired. Due to the compensatory function of the heart, cardiac ejection can still be maintained in a normal state at this time to keep heart function relatively normal.

High altitude exposure can increase pulmonary artery pressure and decrease right heart function (28). The main mechanism of the increase in pulmonary artery pressure caused by acute altitude exposure is pulmonary vasoconstriction (29). If the pulmonary artery pressure rises sharply, it will cause high altitude pulmonary edema (HAPE) (30). However, the pulmonary hypertension caused by chronic altitude exposure is mainly due to changes in the structure of the pulmonary blood vessels, such as the hyperplasia of the middle pulmonary artery, etc. (31). Pulmonary vessel remodeling can cause an irreversible increase in pulmonary artery pressure and right ventricular afterload, which will lead to right ventricular hypertrophy and decline in function (32). For the changes in cardiac structure and function caused by hypoxic pulmonary hypertension, current treatment strategies are mainly focused on reducing pulmonary vasoconstriction and remodeling, and improving symptoms $(33,34)$. Including diuretics to improve right heart failure, anticoagulation therapy to reduce the hazard of thrombosis, oxygen therapy to treat hypoxemia $(35,36)$, selective or non-selective vasodilators such as calcium antagonists, prostacyclin, etc., which have a relaxing effect on vascular smooth muscle cells, inhibits the proliferation of vascular smooth muscle, reverses vascular remodeling, and reduces vascular endothelial damage (33). In addition, a small amount of NO can reduce pulmonary artery pressure and increase oxygenation index. Finally, improving lifestyle and Chinese medicine treatment are also very good choices.

In this study, ultra-high-field magnetic resonance was used to analyze the changes in the structure and function of the right ventricle in rats under chronic hypoxic conditions at high altitude, and pathology and imaging have been combined to further explain the impact of chronic hypoxia at high altitude on the structure and function of the right ventricle.

\section{Strengths and weaknesses}

This experiment was based on the true reproduction of a high-altitude environment with low pressure and hypoxia, and changes in the structure and function of the rat heart under a high-altitude environment with low pressure and hypoxia had been observed. The parameters that have been obtained were representative of the changes in heart structure and function in the natural environment found in plateau regions.

\section{Limitations}

The study was in the stage of exploration and learning. Due to the difficulty of magnetic resonance scanning and postprocessing technology, the scanning time arrangement of plain group rats was unreasonable and the sample size was small. Due to the short exposure time at high altitude, the pathological and physiological evolution of rat right ventricle cannot be studied in stages, which can be used as the research direction in the future.

In this study a 7.0 $\mathrm{T}$ ultra-high-field MRI was used to scan the right ventricle of SD rats raised at different altitudes, and the differences in the partial structure and function parameters of the right ventricle that have been obtained are statistically significant, providing a solid basis for future clinical research. In addition, the study also provided a reference for animal experimental models of high-altitude heart disease in the natural environment of plateau areas.

\section{Acknowledgments}

Funding: Supported by the Qinghai Provincial Health 
Committee Guiding Program Project (2020-wjzdx-04) and the Qinghai Provincial Department of Science and Technology Basic Research Project (2021-ZJ-732).

\section{Footnote}

Reporting Checklist: The authors have completed the ARRIVE reporting checklist. Available at https://dx.doi. org/10.21037/atm-21-5078

Data Sharing Statement: Available at https://dx.doi. org/10.21037/atm-21-5078

Conflicts of Interest: All authors have completed the ICMJE uniform disclosure form (available at https://dx.doi. org/10.21037/atm-21-5078). The authors report funding support from the Qinghai Provincial Health Committee Guiding Program Project (2020-wjzdx-04) and the Qinghai Provincial Department of Science and Technology Basic Research Project (2021-ZJ-732), payments were made to the institution. The authors have no other conflicts of interest to declare.

Ethical Statement: The authors are accountable for all aspects of the work in ensuring that questions related to the accuracy or integrity of any part of the work are appropriately investigated and resolved. The disposal of animals during the experiment complied with the Pain Management Standards in the Eighth Edition of "Guidelines for the Care and Use of Laboratory Animals". Experiments were performed under a project license [No. SYXK (Qing) 2019-0001] granted by the ethics committee of Qinghai Provincial Department of Science and Technology, in compliance with the Drug Inspection and Testing in Qinghai Province institutional guidelines for the care and use of animals.

Open Access Statement: This is an Open Access article distributed in accordance with the Creative Commons Attribution-NonCommercial-NoDerivs 4.0 International License (CC BY-NC-ND 4.0), which permits the noncommercial replication and distribution of the article with the strict proviso that no changes or edits are made and the original work is properly cited (including links to both the formal publication through the relevant DOI and the license). See: https://creativecommons.org/licenses/by-nc-nd/4.0/.

\section{References}

1. Hartman-Ksycińska A, Kluz-Zawadzka J, Lewandowski B. High altitude illness. Przegl Epidemiol 2016;70:490-9.

2. Meier D, Collet TH, Locatelli I, et al. Does This Patient Have Acute Mountain Sickness?: The Rational Clinical Examination Systematic Review. JAMA 2017;318:1810-9.

3. Sakai A, Matsumoto T, Saitoh M, et al. Cardiopulmonary hemodynamics of blue-sheep, Pseudois nayaur, as highaltitude adapted mammals. Jpn J Physiol 2003;53:377-84.

4. Nepal O, Pokharel BR, Khanal K, et al. Relationship between arterial oxygen saturation and hematocrit, and effect of slow deep breathing on oxygen saturation in Himalayan high altitude populations. Kathmandu Univ Med J (KUMJ) 2012;10:30-4.

5. Altland PD, Highman B. Effects of polycythemia and altitude hypoxia on rat heart and exercise tolerance. Am J Physiol 1971;221:388-93.

6. Singh M, Shukla D, Thomas P, et al. Hypoxic preconditioning facilitates acclimatization to hypobaric hypoxia in rat heart. J Pharm Pharmacol 2010;62:1729-39.

7. Cohn JN, Ferrari R, Sharpe N. Cardiac remodeling-concepts and clinical implications: a consensus paper from an international forum on cardiac remodeling. Behalf of an International Forum on Cardiac Remodeling. J Am Coll Cardiol 2000;35:569-82.

8. Manabe I, Shindo T, Nagai R. Gene expression in fibroblasts and fibrosis: involvement in cardiac hypertrophy. Circ Res 2002;91:1103-13.

9. Norman JT, Clark IM, Garcia PL. Hypoxia promotes fibrogenesis in human renal fibroblasts. Kidney Int 2000;58:2351-66.

10. Stenmark KR, Gerasimovskaya E, Nemenoff RA, et al. Hypoxic activation of adventitial fibroblasts: role in vascular remodeling. Chest 2002;122:326S-34S.

11. Tomasek JJ, Gabbiani G, Hinz B, et al. Myofibroblasts and mechano-regulation of connective tissue remodelling. Nat Rev Mol Cell Biol 2002;3:349-63.

12. Palmer BF, Clegg DJ. Oxygen sensing and metabolic homeostasis. Mol Cell Endocrinol 2014;397:51-8.

13. Tucker CE, James WE, Berry MA, et al. Depressed myocardial function in the goat at high altitude. J Appl Physiol 1976;41:356-61.

14. Soltysinska E, Olesen SP, Osadchii OE. Myocardial structural, contractile and electrophysiological changes 


\section{Page 10 of 10}

in the guinea-pig heart failure model induced by chronic sympathetic activation. Exp Physiol 2011;96:647-63.

15. Naeije R. Physiological adaptation of the cardiovascular system to high altitude. Prog Cardiovasc Dis 2010;52:456-66.

16. Nagueh SF, Smiseth OA, Appleton CP, et al. Recommendations for the Evaluation of Left Ventricular Diastolic Function by Echocardiography: An Update from the American Society of Echocardiography and the European Association of Cardiovascular Imaging. Eur Heart J Cardiovasc Imaging 2016;17:1321-60.

17. Karamitsos TD, Dall'Armellina E, Choudhury RP, et al. Ischemic heart disease: comprehensive evaluation by cardiovascular magnetic resonance. Am Heart J 2011;162:16-30.

18. Salerno M, Sharif B, Arheden H, et al. Recent Advances in Cardiovascular Magnetic Resonance: Techniques and Applications. Circ Cardiovasc Imaging 2017;10:e003951.

19. Carbone L. Pain management standards in the eighth edition of the Guide for the Care and Use of Laboratory Animals. J Am Assoc Lab Anim Sci 2012;51:322-8.

20. Tchir JD, Acker JP, Holovati JL. Rejuvenation of ATP during storage does not reverse effects of the hypothermic storage lesion. Transfusion 2013;53:3184-91.

21. Hackett PH, Roach RC. High-altitude illness. N Engl J Med 2001;345:107-14.

22. Zhao SY, Wu XM, Guo YM, et al. Study of blood oxygen saturation, heart rate changes and plateau reaction of the Antarctic Kunlun station investigation team in different plateau environments. Zhonghua Yi Xue Za Zhi 2013;93:1743-5.

23. Tekin D, Dursun AD, Baştuğ M, et al. The effects of acute and intermittent hypoxia on the expressions of HIF- $1 \alpha$ and VEGF in the left and right ventricles of the rabbit heart. Anadolu Kardiyol Derg 2011;11:379-85.

24. Liu H, Yang Q, Radhakrishnan K, et al. Role of VEGF and tissue hypoxia in patterning of neural and vascular cells recruited to the embryonic heart. Dev Dyn 2009;238:2760-9.

Cite this article as: Sun Y, Zhang C, Tian D, Bai J, Li Y, Yu X, Yang J, Wang X, Dong Y, Yang M, Kang Z, Zhang Q, Gao F. Application of 7.0 $\mathrm{T}$ ultra-high-field MRI in evaluating the structure and function of the right ventricle of the heart in rats under a chronic hypoxic environment at high altitude. Ann Transl Med 2021;9(20):1585. doi: 10.21037/atm-21-5078

\section{Sun et al. Structure and function of the right heart in plateau rats}

25. Yang SL, Zhang H, Liu HL. High Altitude Heart Disease. Chin J Cardi Ovasc Reh Abil Med 2008;17:199-200.

26. Sridharan V, Guichard J, Bailey RM, et al. The prolyl hydroxylase oxygen-sensing pathway is cytoprotective and allows maintenance of mitochondrial membrane potential during metabolic inhibition. Am J Physiol Cell Physiol 2007;292:C719-28.

27. Nakada Y, Canseco DC, Thet S, et al. Hypoxia induces heart regeneration in adult mice. Nature 2017;541:222-7.

28. Reichenberger F, Kohstall M G, Seeger T, et al. Effect of sildenafil on hypoxia-induced changes in pulmonary circulation and right ventricular function. Respiratory Physiology \& Neurobiology 2007;159:196-201.

29. Mark EA. Hypoxic pulmonary vasoconstriction. Physiological Reviews 2012;88:367-520.

30. Grünig E, Mereles D, Hildebrandt W, et al. Stress Doppler echocardiography for identification of susceptibility to high altitude pulmonary edema. J Am Coll Cardiol 2000;35:980-7.

31. Mauban JR, Remillard CV, Yuan JX. Hypoxic pulmonary vasoconstriction: role of ion channels. J Appl Physiol (1985) 2005;98:415-420.

32. Xu XQ, Jing ZC. High-altitude pulmonary hypertension. Eur Respir Rev 2009;18:13-7.

33. Frumkin LR. The pharmacological treatment of pulmonary arterial hypertension. Pharmacol Rev 2012;64:583-620.

34. O'Callaghan DS, Savale L, Montani D, et al. Treatment of pulmonary arterial hypertension with targeted therapies. Nat Rev Cardiol 2011;8:526-38.

35. Simonneau G, Robbins IM, Beghetti M, et al. Updated clinical classification of pulmonary hypertension. J Am Coll Cardiol 2009;54:S43-S54.

36. Galiè N, Seeger W, Naeije R, et al. Comparative analysis of clinical trials and evidence-based treatment algorithm in pulmonary arterial hypertension. J Am Coll Cardiol 2004;43:81S-88S.

(English Language Editor: E. Davis) 